\title{
A New Solution Approach to Polynomial LPV System Analysis and Synthesis
}

\author{
Fen Wu and Stephen Prajna
}

\begin{abstract}
Based on Sum-of-Squares (SOS) decomposition, we propose a new solution approach for polynomial LPV system analysis and control synthesis problems. Instead of solving matrix variables over a positive definite cone, the SOS approach tries to find a suitable decomposition to verify the positiveness of given polynomials. The complexity of the SOS-based numerical method is polynomial of the problem size. This approach also leads to more accurate solutions to LPV systems than most existing relaxation methods. Several examples have been used to demonstrate benefits of the SOSbased solution approach.
\end{abstract}

\section{INTRODUCTION}

The study of linear parameter-varying (LPV) systems is motivated by the gain scheduling control design methodology [20], [19]. This class of systems is different from its standard linear time-varying counterpart due to the causal dependence of its controller gains on the variation of the plant dynamics. The LPV formulation allows for general parameter-dependence other than usual continuity requirement. LPV control theory is advantageous because it provides stability and performance guarantee over wide range of changing parameters. The use of a single or parameter-dependent quadratic Lyapunov functions in the analysis and control design for parameter-dependent plants has been studied in a robust control framework [3], [25], [26]. Whereas the analysis test in [3] introduced potential conservatism by measuring performance against arbitrarily fast variations in scheduling parameters, known bounds on the rate of parameter variation were incorporated into the analysis conditions in [25], [26]. In general, the solution to the LPV control analysis and synthesis problems is formulated as a parameter-dependent linear matrix inequalities (LMIs), which is a special type of convex optimization problem.

Parameterized linear matrix inequalities (PLMIs), that is LMIs depending on a parameter confined to a compact set frequently arise in both analysis and synthesis problems of LPV control theory. PLMIs are equivalent to an infinite family of LMI constraints and consequently are very hard to solve numerically. Except for some special cases (affine parameter dependency), infinite number of computation is typically required to solve the parameter-dependent LMI directly. Although a brutal force gridding method [25] can be used to divide the parameter space and renders

F. Wu, Corresponding author, Dept. of Mechanical and Aerospace Engineering, North Carolina State University, Raleigh, NC 27695, fwu@eos.ncsu . edu

S. Prajna, Control and Dynamical Systems, California Institute of Technology, Pasadena, CA 91125, prajna@cds.caltech.edu the infinite-dimensional optimization problem finite, the number of resulting LMI constraints grows rapidly as the number of parameters increases. Moreover, this method only provides an approximated solution which satisfies the LMI constraints at gridding points in the parameter space. The result from finite gridding points is, thus, unreliable. On the other hand, alternative approaches have been actively sought to turn PLMIs into a standard LMI problem by constructing their relaxation forms. In the case of LPV systems depending affinely on the scheduling parameter, vertex method was considered in [3] to determine constant Lyapunov functions satisfying affine parameter-dependent LMI. The solution is exact but it prevents the possibility of using parameter-dependent Lyapunov functions. In [26], [2], convex covering techniques were applied to parameterdependent LMIs to obtain parameter-dependent solutions. However, these methods often require large division numbers to achieve accurate results. Multi-convexity properties was imposed in [6] to provide a finite set of LMIs in solving PLMI problems. In the same vein, recent research [22] explored different relaxation approaches such as separate and difference of convexity to render vertex-type solvability conditions for PLMIs. The relaxation methods involve a finite number of LMIs, which grows exponentially with the number of vertices. Nevertheless, such approaches are potentially conservative as only upper bounds of the original problems are obtained.

Closely related to LPV system descriptions, the switched or hybrid systems have their dynamics described by a set of continuous time differential equations in conjunction with a discrete event process. Due to their wide applications in adaptive control, air-traffic management, and reconfigurable control, the study of switched and hybrid systems have become an important research area in recent years. As shown in [10], the dynamic behavior of switched and hybrid systems is much more complicated than either continuous or discrete dynamics. One way of proving stability of these complex systems is using piecewise continuous Lyapunov functions [16], [9], [17]. Other approaches considered discontinuous Lyapunov functions to verify the stability property of switched and hybrid systems, and can be found in [15], [5]. However, most of the work are focused on switched/hybrid systems with each subsystems described by LTI dynamics with the exception of [17], in which nonlinear subsystems are considered. Moreover, the performance issue of switched/hybrid systems has not been adequately addressed. Recently, the analysis and control of switched LPV systems have been studied in [11], and 
later generalized in [12] by introducing average dwell-time switching logic [8].

In this research, we will study efficient numerical method to solve parameter-dependent LMIs associated with LPV analysis and synthesis problems. The state-space matrices of LPV systems under consideration are polynomial functions of the scheduling parameters, which can not be solved accurately using available techniques. Our proposed solution approach is based on SOS decomposition and semidefinite programming, which have its computational complexity in polynomial time of the problem size. Different from previous convexification methods, the SOS-based approach generalizes the well-known $S$-procedure by searching for parameter-dependent multipliers. Generally speaking, the SOS-based approach will provide less conservative results than most available methods. The SOS solution approach is also beneficial to handle equality-type constraints, as will be shown in a hybrid LPV control design problem. The efficacy of the SOS based solution approach will be demonstrated by several examples.

The notation is standard. $\mathbf{R}$ stands for the set of real numbers and $\mathbf{R}_{+}$for the non-negative real numbers. $\mathbf{R}^{m \times n}$ is the set of real $m \times n$ matrices. The transpose of a real matrix $M$ is denoted by $M^{T}$. We use $\mathbf{S}^{n \times n}$ to denote real, symmetric $n \times n$ matrices, and $\mathrm{S}_{+}^{n \times n}$ for positive definite matrices. If $M \in \mathbf{S}^{n \times n}$, then $M>0(M \geq$ 0 ) indicates that $M$ is a positive definite (positive semidefinite) matrix and $M<0(M \leq 0)$ denotes a negative definite (negative semi-definite) matrix. For any matrix $P$, $\operatorname{Ker}(P)$ stands for the orthogonal complement of matrix $P$. A block diagonal matrix with matrices $X_{1}, \cdots, X_{p}$ on its main diagonal is denoted $\operatorname{diag}\left\{X_{1}, \cdots, X_{p}\right\}$. For $x \in \mathbf{R}^{n}$, its norm is defined as $\|x\|:=\left(x^{T} x\right)^{\frac{1}{2}}$. The space of square integrable functions is denoted by $\mathcal{L}_{2}$, that is, for any $u \in \mathcal{L}_{2},\|u\|_{2}:=\left[\int_{0}^{\infty} u^{T}(t) u(t) d t\right]^{\frac{1}{2}}$ is finite. The spaces of continuously differentiable functions will be denoted by $\mathcal{C}^{1}$ and the corresponding norm is $\|\phi\|=\sup _{t}\|\phi(t)\|$.

\section{SOS DECOMPOSITION TECHNIQUE}

A multivariate polynomial $f\left(x_{1}, \cdots, x_{n}\right)$ is an SOS, if there exist polynomials $f_{1}(x), \cdots, f_{m}(x)$ such that $f(x)=$ $\sum_{i=1}^{m} f_{i}^{2}(x)$. This clearly implies $f(x) \geq 0$ for any $x \in \mathbf{R}^{n}$. SOS decomposition provides a sufficient condition for nonnegativity of a multivariate polynomial, and is equivalent to the existence of a positive-semidefinite matrix $Q$ and a properly chosen vector of monomials $Z(x)$ such that $f(x)=Z^{T}(x) Q Z(x)$. While being stricter, the condition that $f(x)$ is SOS is computationally much more tractable than positivity. At the same time, practical experience indicates that replacing non-negativity by the SOS characterization often leads to the exact solution. For example, when $f\left(x_{1}, \cdots, x_{n}\right)$ is a quadratic function of its variables, its non-negativity is equivalent to the existence of an SOS decomposition [14].

The main advantages of SOS decomposition are the resulting computational tractability and the algorithmic character of the solution procedure [14]. This could help to provide coherent methodology of synthesizing Lyapunov functions for nonlinear systems. In addition, the importance of SOS technique also lies in its ability to provide tractable relaxations for many difficult optimization problems. As is known, many standard optimization problems (such as dissipativity and passivity) in system and control theory are in the form of indefinite quadratic problems [24]. The maximization of these functionals subject to indefinite quadratic constraints is often computationally complicated, sometimes even NP-hard (i.e. non-existence of a polynomial-time algorithm to solve the problem).

The SOS technique is a major breakthrough in system and control theory, as it handles polynomial nonlinearities exactly and solves the problem algorithmically. However, its applications to system and control field have not been fully explored.

Following the convention of [18], a multiplier is called SOS multiplier if itself is in SOS form. A general multiplier is usually referred as polynomial multiplier.

\section{POLYNOMIÁl LPV SySTEM ANALYSIS AND CONTROL SYNTHESIS}

Consider an LPV, system with polynomial parameter dependency

$$
\left[\begin{array}{l}
\dot{x} \\
e
\end{array}\right] \vdots\left[\begin{array}{ll}
A(\rho) & B(\rho) \\
C(\rho) & D(\rho)
\end{array}\right]\left[\begin{array}{l}
x \\
d
\end{array}\right]
$$

where $x, \dot{x} \in \mathbf{R}^{n}, d \in \mathbf{R}^{n_{d}}, e \in \mathbf{R}^{n_{e}}$. All the statespace matrices are polynomial functions of the scheduling parameters and have compatible dimensions. The polynomial LPV systems are not very restrictive because any continuous functions can be approximated adequately by sufficient large order of polynomials. Moreover, some types of rational and nonlinear functions can also be described by polynomial functions with the introduction of auxiliary variables [13]. It is assumed that the vector-valued parameter $\rho$ evolves continuously over time and its range is limited to a set $\mathcal{P} \subset \mathbf{R}^{s}$. In addition, its time derivative is bounded and satisfies the constraint $\underline{\nu}_{i} \leq \dot{\rho}_{i} \leq \bar{\nu}_{i}, i=1,2, \cdots, s$. For notational purposes, denote

$$
\begin{aligned}
& \mathcal{P}=\left\{\rho \in \mathbf{R}^{s}:{ }^{\prime} f_{i}(\rho) \geq 0, i=1,2, \cdots, r_{f}\right\} \\
& \mathcal{V}=\left\{\nu \in \mathbf{R}^{s}: \underline{\nu}_{i} \leq \nu_{i} \leq \bar{\nu}_{i}, i=1,2, \cdots, s\right\}
\end{aligned}
$$

where $f_{i}$ are polynomial functions of $\rho$ which define the boundary of parameter set $\mathcal{P}$. At each time instant $t$, the parameter and its derivative $(\rho(t), \dot{\rho}(t))$ are assumed measurable in real-time. Given the sets $\mathcal{P}$ and $\mathcal{V}$, one can define the parameter $\nu$-variation set as

$$
\mathcal{F}_{\mathcal{P}}^{\nu}=\left\{\rho \in \mathcal{C}^{1}\left(\mathbf{R}_{+}, \mathbf{R}^{s}\right): \rho(t) \in \mathcal{P}, \dot{\rho}(t) \in \mathcal{V}, \forall t \geq 0\right\}
$$

The set $\mathcal{F}_{\mathcal{P}}^{\nu}$ specifies the set of all allowable parameter trajectories.

The stability and achievable induced $\mathcal{L}_{2}$ performance of the LPV systems can be established by solving the 
following LMI

$$
\begin{aligned}
& M(\rho, \nu)=\left[\begin{array}{c}
A^{T}(\rho) X(\rho)+X(\rho) A(\rho)+\sum_{i=1}^{s} \nu_{i} \frac{\partial X}{\partial \rho_{i}} \\
B^{T}(\rho) X(\rho) \\
C(\rho)
\end{array}\right. \\
& \left.\begin{array}{cc}
X(\rho) B(\rho) & C^{T}(\rho) \\
-\gamma I & D^{T}(\rho) \\
D(\rho) & -\gamma I
\end{array}\right]<0
\end{aligned}
$$

for all $(\rho, \nu) \in \mathcal{P} \times \mathcal{V}$. The solvability condition (2) is clearly infinite-dimensional, as is the solution space. The major difficulty in solving LPV analysis problem lies in how the condition (2) will be verified over the entire parameter space. To approximate the infinite-dimensional functional space, we often restrict the search of parameterdependent Lyapunov function to be polynomial functions of scheduling parameters. For example, when two parameters are involved, one may choose

$$
X(\rho)=\sum_{i=0}^{N} \sum_{j=0}^{i} \rho_{1}^{i} \rho_{2}^{j} X_{i j}
$$

where $X_{i j}$ are new optimization variables to be determined. This corresponds to a parameter-dependent Lyapunov function in quadratic form with $N^{\text {th }}$-order parameter dependency. After such a parameterization, the LPV analysis condition can be solved using an ad-hoc gridding method over parameter space. However, the resulting $\gamma$ from gridding method only provides a lower bound of the actual performance in general. On the other hand, either multiconvexity/difference of convexification or convex covering methods [6], [22], [26], [2] can also be employed to solve the above parameter-dependent LMI. After all, the bounds derived from the above approaches are either tooconservative or overly optimistic in the solutions.

As argued above, it is a challenging problem to compute parameter-dependent Lyapunov functions constrained by LMIs, since such functions are non-quadratic polynomials of the parameter variables. In this paper, we will take advantage of the computational tractability of the sum of squares (SOS) decomposition to derive solvability conditions for LPV systems. For this purpose, note that both conditions of $V(x, \rho)$ positive definite and $\frac{\partial V}{\partial x} \dot{x}$ negative definite can be formulated as SOS problems and solved using semidefinite programming. The following result determines the stability and performance of polynomial LPV systems using a parameter-dependent Lyapunov function, which also has polynomial parameter dependency on $\rho$.

Theorem 1: Given the compact sets $\mathcal{P}$ and $\mathcal{V}$, if there exist polynomial matrix function $X: \mathbf{R}^{s} \rightarrow \mathcal{S}_{+}^{n \times n}$ and SOS multipliers $m_{i}(z, \rho, \nu), n_{i}(z, \rho)$, such that

$$
\begin{array}{r}
-z^{T} M(\rho, \nu) z-\epsilon z^{T} z-\sum_{i=1}^{r_{f}} m_{i}(z, \rho, \nu) f_{i}(\rho) \\
-\sum_{i=1}^{s} n_{i}(z, \rho)\left(\nu_{i}-\underline{\nu_{i}}\right)\left(\bar{\nu}_{i}-\nu_{i}\right) \text { is SOS }
\end{array}
$$

for any vector $z$ and a small number $\epsilon>0$, then the LPV system (1) is exponentially stable. In addition, if $d \in \mathcal{L}_{2}$ and $x(0)=0$, then $\|e\|_{2} \leq \gamma\|d\|_{2}$.

To ensure positive definiteness instead of just positive semi-definiteness, we often need to add $-\epsilon z^{T} z$ to the SOS condition (3), where $\epsilon$ is a small positive number. Also note that the parameter derivative $\dot{\rho}$ has been treated as an independent SOS variable in condition (3). This will avoid checking vertex points of the polytope $\mathcal{V}$. Unlike existing numerical algorithms for parameter-dependent LMIs, the computational cost of SOS based approach is fixed for a given LPV system. Specifically, this approach has polynomial-time computational complexity.

Next, we consider an open-loop LPV system with polynomial parameter dependency

$$
\left[\begin{array}{l}
\dot{x} \\
e \\
y
\end{array}\right]=\left[\begin{array}{ccc}
A(\rho) & B_{1}(\rho) & B_{2} \\
C_{1}(\rho) & D_{11}(\rho) & D_{12} \\
C_{2} & D_{21} & 0
\end{array}\right]\left[\begin{array}{l}
x \\
d \\
u
\end{array}\right]
$$

where $\rho \in \mathcal{F}_{\mathcal{P}}^{\nu}, u \in \mathbf{R}^{n_{u}}$ and $y \in \mathbf{R}^{n_{y}}$. Again, we assume that all matrix valued state-space data are polynomial functions with appropriate dimensions. For simplicity, we also assume that

(A1) $\left(A(\rho), B_{2}, C_{2}\right)$ is parameter-dependent stabilizable and detectable for all $\rho$,

(A2) the matrices $\left[\begin{array}{ll}B_{2}^{T} & D_{12}^{T}\end{array}\right]$ and $\left[\begin{array}{ll}C_{2} & D_{21}\end{array}\right]$ have full row ranks.

We would like to design a LPV controller $K_{\mathcal{P}}$ in the form of

$$
\left[\begin{array}{c}
\dot{x}_{k} \\
u
\end{array}\right]=\left[\begin{array}{ll}
A_{k}(\rho, \dot{\rho}) & B_{k}(\rho, \dot{\rho}) \\
C_{k}(\rho, \dot{\rho}) & D_{k}(\rho, \dot{\rho})
\end{array}\right]\left[\begin{array}{c}
x_{k} \\
y
\end{array}\right]
$$

to stabilize the open-loop LPV system (4) with induced $\mathcal{L}_{2}$ norm of the closed-loop system minimized. Note that the controller is scheduled by the parameter $\rho$ and its derivative $\dot{\rho}$.

For constant matrices $\left[\begin{array}{ll}B_{2}^{T} & D_{12}^{T}\end{array}\right]$ and $\left[\begin{array}{ll}C_{2} & D_{21}\end{array}\right]$, the well-known LPV synthesis condition is given by [25]

$$
\begin{aligned}
& Q_{R}(\rho, \nu)=[\star]^{T}\left[\begin{array}{c}
A(\rho) R(\rho)+R(\rho) A^{T}(\rho) \\
-\sum_{i=1}^{s} \nu_{i} \frac{\partial R}{\partial \rho_{i}} \\
C_{1}(\rho) R(\rho)
\end{array}\right\} \\
& \left.\begin{array}{c|c}
R C_{1}^{T}(\rho) & B_{1}(\rho) \\
-\gamma I & D_{11}(\rho) \\
\hline D_{11}^{T}(\rho) & -\gamma I
\end{array}\right]\left[\begin{array}{cc}
\mathcal{N}_{R} & 0 \\
0 & I
\end{array}\right]<0 \\
& Q_{S}(\rho, \nu)=[\star]^{T}\left[\begin{array}{c}
\left\{\begin{array}{c}
A^{T}(\rho) S(\rho)+S(\rho) A(\rho) \\
+\sum_{i=1}^{s} \nu_{i} \frac{\partial S}{\partial \rho_{i}} \\
B_{1}^{T}(\rho) S(\rho)
\end{array}\right. \\
C_{1}(\rho)
\end{array}\right\} \\
& \left.\begin{array}{c|c}
S B_{1}(\rho) & C_{1}^{T}(\rho) \\
-\gamma I & D_{11}^{T}(\rho) \\
\hline D_{11}(\rho) & -\gamma I
\end{array}\right]\left[\begin{array}{cc}
\mathcal{N}_{S} & 0 \\
0 & I
\end{array}\right]<0 \\
& {\left[\begin{array}{cc}
R(\rho) & I \\
I & S(\rho)
\end{array}\right] \geq 0}
\end{aligned}
$$


wherc

$$
\mathcal{N}_{R}=\operatorname{Ker}\left[\begin{array}{ll}
B_{2}^{T} & D_{12}^{T}
\end{array}\right], \quad \mathcal{N}_{S}=\operatorname{Ker}\left[\begin{array}{ll}
C_{2} & D_{21}
\end{array}\right]
$$

The above LPV output-feedback synthesis condition can also be solved using SOS decomposition and semidefinite programming. Its SOS-based solution is provided in the following theorem.

Theorem 2: Given a performance level $\gamma>0$, the compact sets $\mathcal{P}, \mathcal{V}$, and the open-loop polynomial LPV system in (4), if there exist polynomial matrix functions $R, S: \mathbf{R}^{s} \rightarrow \mathcal{S}_{+}^{n \times n}$ and SOS multipliers $m_{1 i}(z, \rho, \nu), m_{2 i}(z, \rho, \nu), m_{3 i}(z, \rho), n_{1 i}(z, \rho), n_{2 i}(z, \rho)$ such that

$$
\begin{gathered}
-z_{1}^{T} Q_{R}(\rho, \nu) z_{1}-\epsilon_{1} z_{1}^{T} z_{1}-\sum_{i=1}^{r_{f}} m_{1 i}\left(z_{1}, \rho, \nu\right) f_{i}(\rho) \\
-\sum_{i=1}^{s} n_{1 i}\left(z_{1}, \rho\right)\left(\nu_{i}-\underline{\nu}_{i}\right)\left(\bar{\nu}_{i}-\nu_{i}\right) \text { is } \operatorname{SOS} \\
-z_{2}^{T} Q_{S}(\rho, \nu) z_{2}-\epsilon_{2} z_{2}^{T} z_{2}-\sum_{i=1}^{r_{f}} m_{2 i}\left(z_{2}, \rho, \nu\right) f_{i}(\rho) \\
-\sum_{i=1}^{s} n_{2 i}\left(z_{2}, \rho\right)\left(\nu_{i}-\underline{\nu}_{i}\right)\left(\bar{\nu}_{i}-\nu_{i}\right) \text { is SOS } \\
z_{3}^{T}\left[\begin{array}{cc}
R(\rho) & I \\
I & S(\rho)
\end{array}\right] z_{3}-\sum_{i=1}^{r_{f}} m_{3 i}\left(z_{3}, \rho\right) f_{i}(\rho) \text { is SOS }
\end{gathered}
$$

for any vectors $z_{1}, z_{2}$ and $z_{3}$ with suitable dimensions and some positive numbers $\epsilon_{1}, \epsilon_{2}$, then there exists an LPV controller $K_{\mathcal{P}}$ that renders the closed-loop LPV system exponentially stable and $\|e\|_{2}<\gamma\|d\|_{2}$.

As mentioned before, the computational complexity of LPV analysis and synthesis conditions based on SOS decomposition is polynomial in time. The derived solvability conditions are generally stricter than the original LMI conditions. However, our experience has shown that the SOS approach often provides less conservative results than other relaxation methods for polynomial LPV systems.

\section{Hybrid LPV State-FEedback CONTROL}

Hybrid LPV control strategy permits using different controllers over different operating ranges. This will lead to relaxed stability condition and provide enhanced design flexibility. In this section, we will consider switching control of LPV systems using multiple state-feedback control laws $u_{i}=r_{i}(\rho) x$.

Consider an open-loop LPV system

$$
\left[\begin{array}{l}
\dot{x} \\
e
\end{array}\right]=\left[\begin{array}{ccc}
A(\rho) & B_{1}(\rho) & B_{2} \\
C_{1}(\rho) & D_{11}(\rho) & D_{12}
\end{array}\right]\left[\begin{array}{l}
x \\
d \\
2 u
\end{array}\right]
$$

where the parameter-dependent matrices functions are polynomials of the scheduling parameter $\rho$. For simplicity, the matrices $B_{2}, D_{12}$ are assumed to be parameter independent. It is also assumed that all the states are available for feedback control usc.
Let $\left\{\mathcal{P}_{i}\right\}_{i=1}^{N}$ be a partition of the parameter set $\mathcal{P}$ as finite number of closed subsets. Each subset $\mathcal{P}_{i}$ is a partial region of the parameter space described by

$$
\mathcal{P}_{i}=\left\{\rho \in \mathbf{R}^{s}: g_{i k}(\rho) \geq 0, k=1,2, \cdots r_{g_{i}}\right\} \subset \mathcal{P}
$$

Thus $\mathcal{P}=\bigcup_{i=1}^{N} \mathcal{P}_{i}$. We also assume that the intersection of any two adjacent subsets is non empty. The switching surfaces for two adjacent parameter subsets $\mathcal{P}_{i}$ and $\mathcal{P}_{j}$ are defined as

$$
\mathcal{S}_{i j}=\left\{\rho \in \mathbf{R}^{s}: h_{i j k}(\rho)=0, k=1,2, \cdots, r_{h_{i j}}\right\}
$$

Note that the surface $\mathcal{S}_{i j}$ specifies the one-directional move from subset $\mathcal{P}_{i}$ to $\mathcal{P}_{j}$. Therefore the sets $\mathcal{S}_{i j}$ and $\mathcal{S}_{j i}$ will specify two different switching surfaces. The functions $g_{i k}, h_{i j k}$ are in their polynomial forms. Due to general form of functions $h_{i j k}$, it can be used to describe nonlinear switching surfaces.

The switching events occur when the trajectory hits one of the switching surfaces $\mathcal{S}_{i j}$. Let $\sigma(0)=i$ if $\rho(0) \in \mathcal{P}_{i}$. For each $t>0$, if $\sigma\left(t^{-}\right)=i$ and $\rho(t) \in \mathcal{P}_{i}$, we keep $\sigma(t)=i$. On the other hand, if $\sigma\left(t^{-}\right)=i$ but $\rho(t) \in \mathcal{P}_{j}$, let $\sigma(t)=j$. Repeating this process, we will generate a piecewise constant signal $\sigma$ which is continuous from the right everywhere. Since $\sigma$ can change its value only after the parameter trajectory has passed through the intersection of adjacent subsets $\mathcal{P}_{i}$ and $\mathcal{P}_{j}$, chattering will be avoided. Moreover, only finite number of switches will happen in any finite time interval due to bounded parameter variation rates. This switching logic is known as "hysteresis switching" [7] and will be used in hybrid LPV control design here. The resulting closed-loop system is a hybrid LPV system, with $\sigma$ being its discrete state. Specifically, the value of $\sigma$ is not only determined by the current valuc of $\rho$ alone, but also depends on the previous values of $\sigma$.

Gencrally speaking, there is no need to associate with each subsystem a global Lyapunov function. In fact, it is often enough to require that each Lyapunov function $V_{i}$ decrease along solutions of the $i$ th subsystem in the region $\mathcal{P}_{i}$ where this system is active. The proposed hysteresis switching control scheme can be thought as a generalization of previous continuous switching approach [11]. However, we do not require continuity of Lyapunov functions across switching surfaces.

Theorem 3: Given a set of scalars $\gamma_{i}$, the parameter set $\mathcal{P}$ and its overlapped partition $\mathcal{P}_{i}, i=1,2, \cdots, N$. There exist switching state-fecdback LPV control laws with associated hysteresis switching logic to render closed-loop exponential stability and achieve performance $\|e\|_{2}<\gamma\|d\|_{2}$ with $\gamma=\max \left\{\gamma_{i}\right\}_{i=1}^{N}$, if there exist parameter-dependent matrix 
functions $R_{i}(\rho)>0$ such that for any $(\rho, \nu) \in \mathcal{P}_{i} \times \mathcal{V}$

$$
\begin{aligned}
& Q_{R i}(\rho, \nu)=[\star]^{T}\left[\begin{array}{c}
\left\{(\rho) R_{i}(\rho)+R_{i}(\rho) A^{T}(\rho)\right. \\
-\sum_{k=1}^{s} \nu_{k} \frac{\partial R_{i}}{\partial \rho_{k}} \\
C_{1}(\rho) R_{i}(\rho)
\end{array}\right\} \\
& \left.\begin{array}{c|c}
R_{i}(\rho) C_{1}^{T} & B_{1}(\rho) \\
-\gamma_{i} I & D_{11}(\rho) \\
\hline D_{11}^{T}(\rho) & -\gamma_{i} I
\end{array}\right]\left[\begin{array}{cc}
\mathcal{N}_{R} & 0 \\
0 & I
\end{array}\right]<0
\end{aligned}
$$

and for any $\rho \in \mathcal{S}_{i j}$

$$
R_{i}(\rho)-R_{j}(\rho) \leq 0
$$

where $\mathcal{N}_{R}=\operatorname{Ker}\left[\begin{array}{ll}B_{2} & D_{12}\end{array}\right]$. Furthermore, the switching state-feedback gains are given by

$$
F_{i}(\rho)=-\left(D_{12}^{T} D_{12}\right)^{-1}\left[\gamma_{i} B_{2}^{T} R_{i}^{-1}(\rho)+D_{12}^{T} C_{1}(\rho)\right]
$$

for $i=1,2, \cdots, N$.

After deriving hybrid LPV state-feedback synthesis condition in LMI form, the SOS decomposition approach can be used to formulate a computable synthesis result. This is given bellow:

Corollary 1: If there exist polynomial matrix functions $R_{i}: \mathbf{R}^{s} \rightarrow \mathbf{S}_{+}^{n \times n}, i=1,2, \cdots N$, SOS multipliers $m_{1 k}(z, \rho, \nu), n_{1 k}(z, \rho)$ and polynomial multipliers $\ell_{i j k}(z, \rho)$ such that

$$
\begin{aligned}
& -z_{1}^{T} Q_{R i}(\rho, \nu) z_{1}-\epsilon z_{1}^{T} z_{1}-\sum_{k=1}^{r_{g_{i}}} m_{i k}\left(z_{1}, \rho, \nu\right) g_{i k}(\rho) \\
& \quad-\sum_{k=1}^{s} n_{1 k}\left(z_{1}, \rho\right)\left(\nu_{k}-\underline{\nu}_{k}\right)\left(\bar{\nu}_{k}-\nu_{k}\right) \text { is SOS } \\
& -z_{2}^{T}\left[R_{i}(\rho)-R_{j}(\rho)\right] z_{2} \\
& +\sum_{k=1}^{r_{h_{i j}}} \ell_{i j k}\left(z_{2}, \rho\right) h_{i j k}(\rho) \text { is SOS }
\end{aligned}
$$

for any vectors $z_{1}$ and $z_{2}$ and a small positive $\epsilon$, then the hybrid LPV system is exponentially stabilizable and the induced $\mathcal{L}_{2}$ norm is bounded by $\gamma=\max \left\{\gamma_{i}\right\}_{i=1}^{N}$.

It is usually difficult to enforce equality constraint like (13) in LMI optimization problem. On the other hand, this type of constraints can be easily handled in SOS tool [18] by introducing polynomial multipliers.

\section{EXAMPLE}

We first consider a gas-turbine engine model described as a linear system with a scalar scheduling parameter [2]

$$
\begin{aligned}
& \dot{x}=\left(A_{0}+A_{1} \theta+A_{2} \theta^{2}\right) x+\left(B_{0}+B_{1} \theta+B_{2} \theta^{2}\right) w \\
& z=C x
\end{aligned}
$$

where $x_{1}, x_{2}$ and $x_{3}$ are the compressor speed, the fan speed and the outlet press, respectively. The scheduling parameter is compressor rotating speed and belongs to $\theta \in[0,1]$. Also

$$
\begin{aligned}
A_{0} & =\left[\begin{array}{ccc}
-4.365 & -0.6723 & -0.3363 \\
7.088 & -6.557 & -4.601 \\
-2.410 & 7.584 & -14.310
\end{array}\right] \\
A_{1} & =\left[\begin{array}{ccc}
-0.56081 & 0.85534 & 0.58923 \\
2.5333 & -1.0398 & -7.7373 \\
3.1917 & 1.7971 & -2.5887
\end{array}\right] \\
A_{2} & =\left[\begin{array}{ccc}
0.66981 & -1.375 & -0.99093 \\
-2.8963 & -1.5292 & 10.516 \\
-3.5777 & 2.8389 & 1.9087
\end{array}\right] \\
B_{0} & =\left[\begin{array}{ccc}
2.374 & 0.7485 \\
1.366 & 3.444 \\
0.9416 & -9.619
\end{array}\right] \\
B_{1} & =\left[\begin{array}{cc}
-0.16023 & -0.35209 \\
0.11622 & -2.4839 \\
-0.11058 & -4.6057
\end{array}\right] \\
B_{2} & =\left[\begin{array}{cc}
0.15623 & 0.13063 \\
-0.49582 & 4.0379 \\
-0.030616 & 0.89473
\end{array}\right] \\
C & =\left[\begin{array}{lll}
0 & 1 & 0 \\
0 & 0 & 1
\end{array}\right] .
\end{aligned}
$$

We would like to evaluate this system's induced $\mathcal{L}_{2}$ performance under different varying parameter assumptions.

Similar to the original paper, we consider three cases:

1) Quadratic Lyapunov function, the optimal induced $\mathcal{L}_{2}$-gain bound is $\gamma=0.9603$.

2) Affine parameter-dependent Lyapunov function with parameter variation rate $\nu \in[-1,1]$, the optimal bound is $\gamma=0.9520$.

3) Quadratic parameter-dependent Lyapunov function with $\nu \in[-10,10]$, the optimal bound is $\gamma=0.9462$.

The bound we have in Case 1 is similar to what the original paper obtained for division number 25 . In cases 2 and 3 our bounds are better than theirs (even when their division number equals to 25). It is interesting to see that higher order parameter-dependent Lyapunov function is very useful to improve the system performance even the parameter variation rates increase from 1.0 to 10 .

The second example is taken from [20] and slightly modified by adding disturbance effect. This plant has poorly damped zeros which vary along the imaginary axis. Its statespace equation is given by

$$
\begin{aligned}
{\left[\begin{array}{l}
\dot{x}_{1} \\
\dot{x}_{2} \\
\dot{x}_{3}
\end{array}\right]=} & {\left[\begin{array}{ccc}
0 & (2-\theta)^{2} & 1+0.5 \theta+(2-\theta)^{2} \\
1 & 0 & 0.2 \\
0 & 0 & 0
\end{array}\right]\left[\begin{array}{l}
x_{1} \\
x_{2} \\
x_{3}
\end{array}\right] } \\
& +\left[\begin{array}{l}
0 \\
1 \\
0
\end{array}\right] d+\left[\begin{array}{l}
0 \\
0 \\
1
\end{array}\right] u
\end{aligned}
$$

where $-1 \leq \theta \leq 1$ and $|\dot{\theta}| \leq 2$. Also the output equation 
is chosen as

$$
e=\left[\begin{array}{lll}
0 & 1 & 1 \\
0 & 0 & 0
\end{array}\right]\left[\begin{array}{l}
x_{1} \\
x_{2} \\
x_{3}
\end{array}\right]+\left[\begin{array}{l}
0 \\
1
\end{array}\right] u
$$

All the states are assumed to be available for feedback control use.

To solve the hybrid state-feedback control problem, we partition the parameter set into either two or four overlapped subsets. That is

- 2 subsets $\theta_{1}=[-1,0.05]$ and $\theta_{2}=[-0.05,1]$.

- 4 subsets $\Theta_{1}=[-1,-0.45], \Theta_{2}=[-0.55,0.05]$, $\Theta_{3}=[-0.05,0.55]$ and $\Theta_{4}=[0.45,1]$.

Then we will solve hybrid LPV control problem by synthesizing multiple Lyapunov functions $V_{i}(x, \theta)=$ $x^{T} R_{i}(\theta)^{-1} x$, where different parameter dependency is considered for matrix function $R(\theta)$. For comparison, we also synthesize a state-feedback control law over the entire parameter set. The performance achieved by non-switching and hybrid LPV state-feedback control is summarized in the following table.

TABLE I

PERFORMANCE USING NON-SWITCHING AND SWITCHED CONTROL STRATEGIES

\begin{tabular}{|c||c|c|c|}
\hline$V(x)$ & 1 subset & 2 subsets & 4 subsets \\
\hline$x^{T} R_{0}^{-1} x$ & 1.3598 & & \\
$x^{T}\left(R_{0}+\theta R_{1}\right)^{-1} x$ & 1.2537 & 1.2168 & 1.1828 \\
$x^{T}\left(R_{0}+\theta R_{1}+\theta^{2} R_{2}\right)^{-1} x$ & 1.1299 & 1.1235 & 1.1186 \\
\hline
\end{tabular}

When higher order parameter-dependent Lyapunov functions are chosen for control synthesis purpose, it is possible to tighten performance of the closed-loop system. Similarly, further partition of the parameter set into smaller regions also helps to improve the performance.

\section{CONCLUDING REMARKS}

In this paper, we have proposed a new solution approach to LPV analysis and synthesis problems for a special class of LPV systems, namely, the LPV systems have polynomial parameter dependency. The proposed solution approach is based on SOS decomposition and is applicable to a large class of LPV systems. This method also provides reliable and less conservative results than most existing relaxation methods. It generalizes the well-known S-procedure to improve LPV analysis and synthesis conditions. Moreover, the SOS-based method is very efficient and has the computational complexity in polynomial time.

If the assumption of constant $B_{2}, D_{12}$ and $C_{2}, D_{21}$ matrices is relaxed to polynomial functions, then the corresponding polynomial LPV analysis and synthesis and hybrid LPV synthesis conditions can be derived using Finsler's lemma. However, the details will not be reported here.

\section{REFERENCES}

[1] P. Apkarian and R.J. Adams, Advanced Gain-Scheduling Techniques for Uncertain Systems, IEEE Trans. Automat. Contr. Tech., vol. 6 , 1997, pp. 21-32.

[2] T. Azuma, R. Watanabe, K. Uchida, and M. Fujita, A New LMI Approach to Analysis of Linear Systems Depending on Scheduling Parameter in Polynomial Forms, Automatiserungstechnik, vol. 48, 2000 , pp. $1-6$.

[3] G. Becker and A.K. Packard, Robust Performance of Linear Parametrically Varying Systems using Parametrically-Dependent Linear Feedback, Syst. Contr. Letts., vol. 23, 1994, pp. 205-215.

[4] S.P. Boyd, L. El Ghaoui, E. Feron, and V. Balakrishnan, Linear Matrix Inequalities in Systems and Control Theory, SIAM, Philadelphia, PA:1994.

[5] M. Branicky, Multiple Lyapunov Functions and Other Analysis Tools for Switched and Hybrid Systems, IEEE Trans. Automat. Contr., vol. 43, 1998, pp. 475-482.

[6] P. Gahinet, P. Apkarian, and M. Chilali, Affine Parameter-Dependent Lyapunov Functions and Real Parametric Uncertainty, IEEE Trans. Automat. Contr, vol. 41, 1996, pp. 436-442.

[7] J. Hespanha, D. Liberzon, and A. Morse, Hysteresis-Based Switching Algorithms for Supervisory Control of Uncertain Systems, Automatica, vol. 39,2003 , pp. 263-272.

[8] 3. Hespanha and A: Morse, "Stability of Switched Systems with Average Dwell-Time," in Proc. IEEE Conf. Dec. Contr, 1999, pp. 2655-2660.

[9] M. Johansson and A. Ranzer, Computation of Piecewise Quadratic Lyapunov Functions, IEEE Trans. Automat. Contr., vol. 43, 1998, pp. 555-559.

[10] D. Liberzon and A. Morse, Basic Problerns in Stability and Design of Switched Systems, IEEE Contr. Syst. Mag., vol. 19, 1999, pp. 59-70.

[11] S. Lim, Analysis and Control of Linear Parameter-Varying Systems, PhD Dissertation, Stanford University, Palo Alto, CA:1999.

[12] S. Lim and K. Chan,' "Analysis of Hybrid Linear Parameter-Varying Systems," in Proc. Amer. Contr. Conf., 2003, pp. 4822-4827.

[13] A. Papachristodoulou and S. Prajna, "On the Construction of Lyapunov Functions using the Sum of Squares Decomposition," in Proc. 2002 IEEE Conf. Dec. Contr, 2002, pp. 3482-3487.

[14] P.A. Parrilo, Structured Semidefinite Programs and Semialgebric Geometry Methods in Robustness and Optimization, California Institute of Technology, Pasadena, CA:2000.

[15] P. Peleties and R. DeCarlo, "Asymptotic Stability of m-Switched Systems using Lyapunov-Like Functions," in Proc. Amer. Contr. Conf., 1991, pp. 1679-1684.

[16] S. Pettersson and B. Lennartson, "Stability and Robustness of Hybrid Systems," in Proc. IEEE Conf. Dec. Contr, 1996, pp. 1202-1207.

[17] S. Prajna and A. Papachristodoulou, "Analysis of Switched and Hybrid Systems-Beyond Piecewise Quadratic Methods," in Proc. Amer. Contr. Conf., 2003, pp. 2779-2784.

[18] S. Prajna, A. Papachristodoulou, and P.A. Parrilo, Sum of Squares Toolbox for MATLAB: User's Guide, ver. 1.0, 2002.

[19] W.J. Rugh, Analytical Framework for Gain Scheduling, IEEE Contr. Sys. Mag., vol. 11, 1991, pp. 74-84.

[20] J.S. Shamma and M. Athans, Analysis of Gain Scheduled Control for Nonlinear Plants, IEEE Trans. Automat. Contr., vol. 35, 1990, pp. 898-907.

[21] J.S. Shamma and M. Athans, Gain Scheduling: Potential Hazards and Possible Remedies, IEEE Contr. Sys. Mag., vol. 12, 1992, pp. 101-107.

[22] H.D. Tuan and P. Apkarian, Relaxations of Parameterized LMIs with Control Applications, Int. J. Robust Non. Contr, vol. 9, 1999, pp. 5984.

[23] Tuan, H.D. and P. Apkarian, Monotonic Relaxations for Robust Control: New Characterizations, IEEE Trans. Automat. Contr, vol. 47, 2002, pp. 378-384:

[24] L. Vandenberghe and S.P. Boyd, Semidefinite Programming, SIAM Review, vol. 38, 1996, pp. 49-95.

[25] F. Wu, X.H. Yang, A.K. Packard, and G. Becker, Induced $\mathcal{L}_{2}$ Norm Control for LPV Systems with Bounded Parameter Variation Rates, Int. J. Robust Non. Contr, vol. 6, 1996, pp. 983-998.

[26] J. Yu and A. Sideris, $\mathcal{H}_{\infty}$ Control with Parametric Lyapunov Functions, Syst. Contr. Letts., vol. 30, 1997, pp. 57-69. 